\title{
Glucose Sensing in Saline Solutions using V-Band Waveguides
}

\author{
Helena Cano García, Ioannis Papadopoulos-Kelidis, \\ Ioannis Sotiriou, George Palikaras, Efthymios Kallos \\ Medical Wireless Sensing Ltd. \\ London, United Kingdom
}

\author{
Panagiotis Kosmas \\ Centre for Telecommunications Research \\ King's College London \\ London, United Kingdom
}

\author{
Clive Parini \\ School of Electronic Engineering and Computer Science \\ Queen Mary University of London \\ London, United Kingdom
}

\begin{abstract}
We present results from an experimental system employed to study the impact of glucose content on measured transmission data. The measurements have been performed at frequencies between $50 \mathrm{GHz}$ and $75 \mathrm{GHz}$ using V-Band waveguides, and the measured samples consisted of saline solutions with glucose concentrations down to $0.025 \mathrm{wt} \%$. The performed transmission measurements show that it is possible to establish an approximately linear relationship between the transmission coefficient and the glucose content in the samples. This linear dependence is also verified via simulations. The results are of interest for developing a miniaturized mobile glucose sensing system.
\end{abstract}

Keywords-glucose sensing; transmission; V-band; waveguides; non-invasive

\section{INTRODUCTION}

Diabetes is a chronic metabolic disorder affecting more than 350 million people worldwide according to the World Health Organization, with the number of people affected set to increase to 550 million by 2030 [1]. In order to tackle the increasing diagnostic needs of diabetes patients, it is necessary to develop affordable and accurate glucose monitoring devices that do not rely on drawing blood and are non-invasive.

The main challenge for non-invasive glucose monitoring lies in the fact that glucose levels in blood vary only in the range of a few milligrams per deciliter or millimole per liter [2]. With this in mind, the motivation behind this paper is the development of a non-invasive glucose monitoring system based on the $50-75 \mathrm{GHz}$ radio wave band. To this end, we present experimental and simulation results from a system that has been developed to demonstrate the feasibility of sensing reliably variations in glucose concentrations within solution of water and salt, by measuring electromagnetic (EM) transmission and reflection data in the $50-75 \mathrm{GHz}$ band.
Previous studies have focused on lower microwave frequencies $(1-20 \mathrm{GHz})$ using different setups to measure the glucose changes in controlled samples, based on the dependence of the sample's permittivity on glucose concentration. For example, microwave cavity experimental setups have been presented in the $2-3 \mathrm{GHz}$ frequency range $[3,4]$, while $1.0-2.5 \mathrm{GHz}[5]$ and $5.0-8.5 \mathrm{GHz}[6]$ ranges have been used in setups with antenna sensors. Open waveguide structures have also been used to measure the effect of varying glucose concentrations on the waveguide's scattering parameters (S-parameters) for frequencies up to $20 \mathrm{GHz}[7]$.

Higher microwave frequencies have also been studied in [8-10], but the measurements in these papers cannot be fully compared to the ones presented in this paper. In particular, [8] uses both reflection and transmission data but focus on non-invasive measurements only up to $40 \mathrm{GHz}$ and higher minimum glucose concentrations, while [9] and [10] cover the frequency range of our study but use reflection data only and also higher minimum glucose concentrations. Thus, the presented measurements are, to the best of our knowledge, the first to show a clear correlation between measured transmission data and varying glucose level for concentrations as low as $0.025 \mathrm{wt} \%(25 \mathrm{mg} / \mathrm{dL})$.

The rest of the paper is organized as follows. Section II presents the preparation procedure of the glucose samples, the setup used in the experiments and the measurement process. Section III presents the results obtained from the experiment and their correlation with the simulations results. Section IV presents the conclusions of the study.

\section{METHODS}

\section{A. Prepared and measured samples}

To perform the experiments, three sets of samples were prepared: i) samples with glucose concentrations ranging 


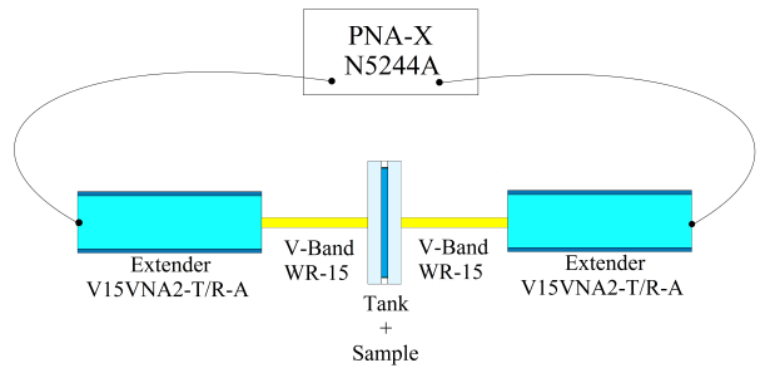

(a)

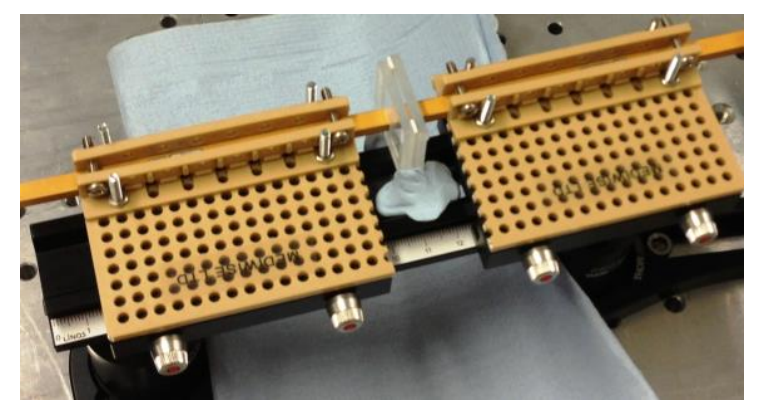

(b)

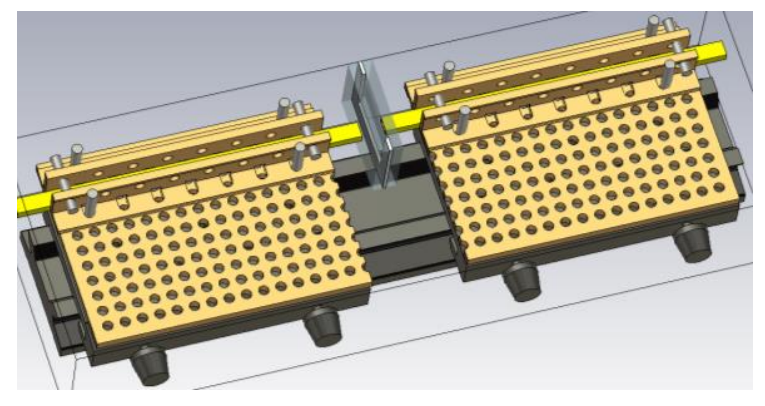

(c)

Figure 1. a) Schematic version of the setup that includes the top view of the tank and the waveguides, the extenders and the PNA-X. b) Experimental setup consisting of a pair of waveguides and their supporting structure; c) Replication of the experimental setup in a simulation environment.

between $0.025 \mathrm{wt} \%(25 \mathrm{mg} / \mathrm{dL})$ and $5 \mathrm{wt} \%(5000 \mathrm{mg} / \mathrm{dL})$ in distilled water; ii) samples with glucose concentrations between $0.025 \mathrm{wt} \%$ and $5 \mathrm{wt} \%$ in saline solution $(0.9 \mathrm{wt} \%$ $\mathrm{NaCl}$ concentration in distilled water), where the saline solution was used in order to obtain more realistic samples, as in $[9,10]$; iii) samples with $\mathrm{NaCl}$ concentrations ranging between $0.05 \mathrm{wt} \%$ and $4 \mathrm{wt} \%$ in $0.9 \mathrm{wt} \%$ glucose concentration in distilled water.

All the samples were prepared following the same procedure. The necessary amount of glucose and salt was calculated using the concentration value (by weight) and the total weight of the sample. First the salt, then the glucose, and distilled water were added to the container. The contents were then mixed using an electric hand mixer (frapediera) until the sample became homogeneous. Subsequently, the sample was stored inside a refrigerator. The samples were brought to room temperature $\left(25.5^{\circ} \mathrm{C}\right)$ before performing the measurements in order to avoid changes in their physical properties due to temperature differences [11].

\section{B. Experimental setup}

To measure the reflection $\left(S_{11}\right)$ and transmission $\left(S_{21}\right)$ coefficients a microwave network analyzer was used, N5244A PNA-X. This model supports a measurement frequency range of $10 \mathrm{MHz}-43.5 \mathrm{GHz}$. In order to reach the desired frequency range of $50-75 \mathrm{GHz}$, a pair of millimeter wave VNA extenders (V15VNA2-T/R-A) were connected between the network analyzer and the measurement setup.

As shown in Fig. 1b, the setup consisted of a supporting structure, two V-band waveguides (WR-15), and an acrylic tank placed between them inside which the liquid samples were placed. The total outer dimensions of the tank were $60 \mathrm{~mm} \times 40 \mathrm{~mm} \times 7 \mathrm{~mm}$. The thickness of the acrylic walls was $3 \mathrm{~mm}$, leaving $1 \mathrm{~mm}$ empty space containing the solutions. Before the measurements, a Thu-Reflect-Line (TRL) calibration [12] was performed using the V11644A calibration kit.

\section{Measurement process}

All measurements were carried out using the same tank, which was placed in a fixed position. The liquid samples were filled and removed from the tank using a syringe. The samples were measured in sequence according to their glucose content, starting with samples with less glucose. This measurement sequence was set in order to minimize the impact of previous samples in each sample that was measured. After each set of samples, distilled water was used to clean the tank.

\section{RESULTS}

\section{A. Experimental results}

The setup shown in Fig. 1b allowed measuring the reflection and transmission coefficients of the three different sets of samples described in Section II-A between $50-75 \mathrm{GHz}$.

Transmission and reflection measurements were performed for all the samples but more sensitivity to glucose variations was observed for transmission data in the frequency ranges $59-64 \mathrm{GHz}$ and $69-73 \mathrm{GHz}$. Samples of the experimental results are presented in Fig. $2 \mathrm{a}$ and Fig. $2 \mathrm{~b}$.

Fig. 2a plots the values of the reflection coefficient at $72.4 \mathrm{GHz}$. In this case, the sensitivity is $0.05 \mathrm{~dB}$ per wt $\%$. Therefore, the paper focuses on the transmission measurements rather than the reflection measurements.

Fig. 2b plots the values of the transmission coefficient at $72.4 \mathrm{GHz}$ for the three different types of samples. As shown in the figure, samples made of different glucose concentrations in water and in saline solution present similar behavior. As the glucose concentration increases the 
transmission coefficient increases approximately linearly as a function of concentration (note the logarithmic scale of the horizontal axis which is necessary to depict clearly the whole range of concentrations) with a slope of $1.1 \mathrm{~dB}$ per $w t \%$. The addition of salt to the samples causes an increase in the transmission amplitude between $0.1 \mathrm{~dB}$ to $0.4 \mathrm{~dB}$, depending on the concentration and the frequency. Furthermore, solutions with varying glucose produce faster changes in the signal than solutions with varying salt, for which the transmission amplitude does not significantly change as the concentration increases. Importantly, these results suggest that the measurements are sensitive to varying glucose concentrations but not to changes in salt concentration.

\section{B. Simulation results}

The approximately linear relationship between the scattering coefficient and the glucose concentration

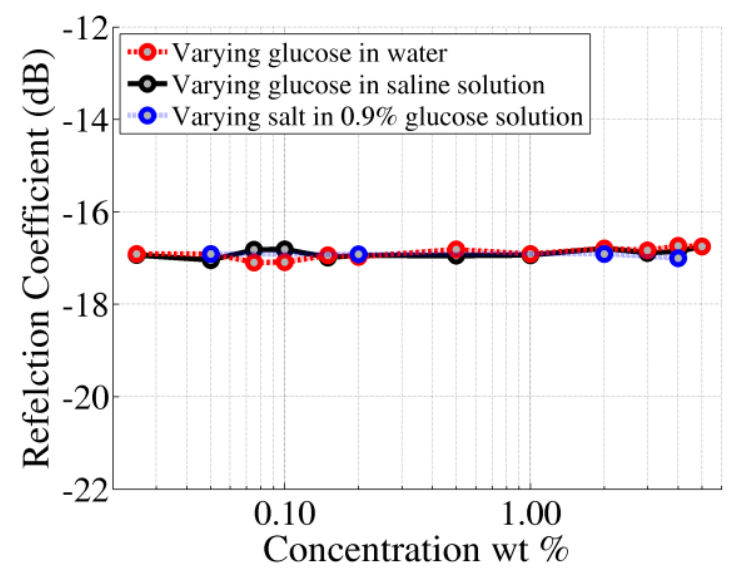

(a)

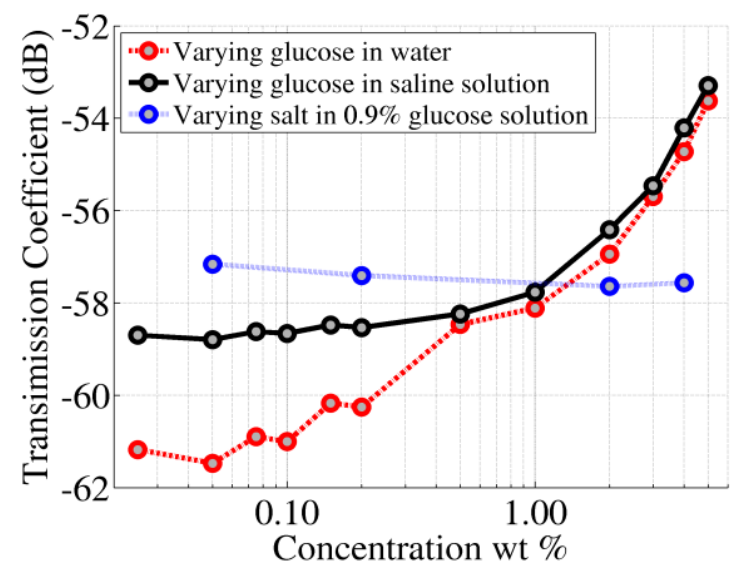

(b)

Figure 2. Experimentally measured $S_{11}$ (a) and $S_{21}$ (b) values at 72.4 $\mathrm{GHz}$ against glucose or salt concentrations (wt\%). The red dash-dot line represents the values for the samples with varying glucose in water only. The black solid line represents the values for the samples with varying glucose in saline solution. The blue dotted line represents the values for the samples with varying salt in $0.9 \mathrm{wt} \%$ glucose concentration in water.

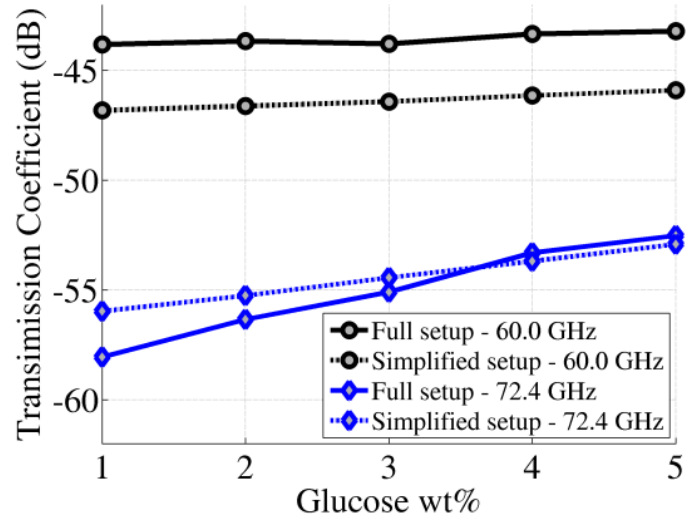

Figure 3. Simulated transmission against glucose concentration for different frequencies. Dotted lines correspond to the results of simulating a simplified part of the setup and solid lines correspond to results obtained simulating the full setup. The trace with circle markers correspond to the transmission coefficietn at $60 \mathrm{GHz}$ and the trace with diamond markers correspond to the transmission coefficient at $72.4 \mathrm{GHz}$.

observed in Fig. 2b was also verified in simulations performed using $\mathrm{CST}^{\mathrm{TM}}$ Microwave Studio software. Two types of simulation results were obtained: the first after simulating the setup shown in Fig. 1c, and the second after simulating a simplified setup consisting of the waveguides and the tank only without the supporting structure. These simulations were performed for glucose concentrations ranging between $1 \mathrm{wt} \%$ and $5 \mathrm{wt} \%$, modeled as dispersive materials that follow a Debye model which was obtained experimentally for each concentration. The results of these simulations are presented in Fig. 3.

Fig. 3 shows the effect in the transmission coefficient due to the components of the supporting structure. This effect is reflected on the changes in the relationship between the transmission coefficient and the glucose content. The full setup results present higher transmission coefficient values than the simplified setup.

The residual of a linear fitted curve for the full setup simulations ranges from $0.25 \mathrm{~dB}$ to $0.49 \mathrm{~dB}$ and the residual of the linear fitted curve for the simplified setup simulations ranges from $0.043 \mathrm{~dB}$ to $0.045 \mathrm{~dB}$. These values suggest that the simplified setup presents a more linear trend than the full setup because the simplified setup does not consider the effects of the supporting structure. The slope for these traces is $0.15 \mathrm{~dB}$ per $\mathrm{wt} \%$ at $60 \mathrm{GHz}$ and $1.41 \mathrm{~dB}$ per wt $\%$ at $72.4 \mathrm{GHz}$ for the full setup; and $0.23 \mathrm{~dB}$ per wt $\%$ at $60 \mathrm{GHz}$ and $0.77 \mathrm{~dB}$ per $\mathrm{wt} \%$ at $72.4 \mathrm{GHz}$ for the simplified setup. The difference between the experimentally measured and simulated slopes is attributed to two factors. First, the simulations were performed using a first order Debye model extrapolated to the $\mathrm{V}$-band frequencies based on dielectric constant measurements at lower frequencies $(500 \mathrm{MHz}-20$ $\mathrm{GHz}$ ). Second, these low frequency measurements used in the simulations were performed at $23{ }^{\circ} \mathrm{C}$, while the experiment took place at $25.5^{\circ} \mathrm{C}$. 


\section{CONCLUSIONS}

A setup consisting of two WR-15 waveguides was used to measure the transmission and reflection coefficient of samples with different types of glucose concentrations in water and saline solutions. These concentrations were in the clinically relevant range between $0.025 \mathrm{wt} \%$ and $5 \mathrm{wt} \%$. The measurements were performed in the frequency range of $50-75 \mathrm{GHz}$ and best results were obtained in the $59-64 \mathrm{GHz}$ and $69-73 \mathrm{GHz}$ ranges.

Glucose concentrations down to $0.025 \mathrm{wt} \%$ were measured with good accuracy $(0.22 \mathrm{~dB}$ per wt $\%)$. This accuracy was also achieved in physiological saline samples (consisting of glucose, salt and water). Higher frequencies presented better accuracy $(0.61 \mathrm{~dB}$ per wt $\%)$ than lower frequencies $(0.33 \mathrm{~dB}$ per wt $\%)$. Finally, an approximately linear relationship between the transmission coefficient and the glucose content was observed in the experimental results and was validated with accurate numerical simulations.

The authors would like to acknowledge the support of the Technology Strategy Board towards the work presented in this paper (grant \# 131618).

\section{REFERENCES}

[1] (2012, May 2014). IDF Diabetes Atlas 2012 (5th ed.).

[2] J. Wang, "Glucose Biosensors: 40 Years of Advances and Challenges," Electroanalysis, vol. 13, pp. 983-988, 2001.

[3] R. Dobson, R. Wu, and P. Callaghan, "Blood glucose monitoring using microwave cavity perturbation," Electronics Letters, vol. 48, pp. 905-906, 2012.

[4] F. Yun, D. Xiaoguang, W. Qing, and W. WeiLian, "Testing glucose concentration in aqueous solution based on microwave cavity perturbation technique," in Biomedical Engineering and Informatics (BMEI), 2010 3rd International Conference on, 2010, pp. 1046-1049.

[5] S. Wiwatwithaya, P. Phasukkit, S. Tungjitkusolmun, and W. Wongtrairat, "Real-time monitoring glucose by used microwave antenna apply to biosensor," in Biomedical Engineering International Conference (BMEiCON), 2011, 2011, pp. 135137.

[6] M. Hofmann, T. Fersch, R. Weigel, G. Fischer, and D. Kissinger, "A novel approach to non-invasive blood glucose measurement based on RF transmission," in Medical Measurements and Applications Proceedings (MeMeA), 2011 IEEE International Workshop on, 2011, pp. 39-42.

[7] M. Hofmann, M. Bloss, R. Weigel, G. Fischer, and D. Kissinger, "Non-invasive glucose monitoring using open electromagnetic waveguides," in Microwave Conference (EuMC), 2012 42nd European, 2012, pp. 546-549.

[8] M. Hofmann, G. Fischer, R. Weigel, and D. Kissinger, "Microwave-Based Noninvasive Concentration Measurements for Biomedical Applications," Microwave Theory and Techniques, IEEE Transactions on, vol. 61, pp. 2195-2204, 2013.

[9] V. V. Meriakri, E. E. Chigrai, I. P. Nikitin, and M. P. Parkhomenko, "Dielectric Properties of Water Solutions with Small Content of Glucose in the Millimeter - Wave Band and the Determination of Glucose in Blood," in Physics and Engineering of Microwaves, Millimeter and Submillimeter Waves and Workshop on Terahertz Technologies, 2007. MSMW '07. The Sixth International Kharkov Symposium on, 2007, pp. 873-875.

[10] V. V. Meriakri, E. E. Chigrai, I. P. Nikitin, and M. P Parkhomenko, "Dielectric properties of glucose solutions in the millimeter-wave range and control of glucose content in blood," in Infrared and Millimeter Waves and 13th International
Conference on Terahertz Electronics, 2005. IRMMW-THz 2005. The Joint 30th International Conference on, 2005, pp. 315-316 vol. 1.

[11] K. Lee, A. Babajanyan, C. Kim, S. Kim, and B. Friedman, "Glucose aqueous solution sensing by a near-field microwave microprobe," Sensors and Actuators A: Physical, vol. 148, pp. 28-32, 2008

[12] D. M. Pozar, Microwave Engineering, Fourth Edition ed., 2012. 